Article

\title{
Asymmetric Friedel-Crafts Alkylation of Indole with Chalcones Catalyzed by Chiral Phosphoric Acids
}

\author{
Arrigo Scettri $^{1}$, Rosaria Villano ${ }^{2}$ and Maria Rosaria Acocella ${ }^{1, *}$
}

1 Dipartimento di Chimica, Università di Salerno, Via Ponte Don Melillo 84084, Fisciano, Salerno, Italy; E-mail: scettri@unisa.it (A.S.)

2 Istituto di Chimica Biomolecolare-CNR Trav. La Crucca3, Reg. Baldinca 07040, Li Punti, Sassari, Italy; E-mail: rosaria.villano@gmail.com (R.V.)

* Author to whom correspondence should be addressed; E-mail: acord78@libero.it; Tel.: +39-089 969 376; Fax: +39-089 969603

Received: 22 July 2009; in revised form: 6 August 2009 / Accepted: 10 August 2009 / Published: 13 August 2009

\begin{abstract}
The reaction of indole with chalcones, to give Michael-type adducts, was found to occur with good efficiency (up to $98 \%$ yield) and moderate enantioselectivity (up to $52 \%$ e.e.) in the presence of a chiral BINOL-based phosphoric acid. Furthermore, the alkylation products can be obtained in much higher e.e.s after one only crystallization.
\end{abstract}

Keywords: asymmetric Friedel-Crafts; indole; chiral phosphoric acids

\section{Introduction}

The indole moiety represents the main structural feature of a variety of unnatural and natural bioactive products, such as the indole alkaloids $[1,2]$. In recent years particular attention has been paid to the enantioselective alkylation of indoles with $\alpha, \beta$-unsaturated carbonyl compounds [3,4] since the corresponding Michael-type adducts could be considered valuable key-intermediates for the construction of chiral indole architectures.

It has to be noted that different approaches have been proposed for the Michael-type Friedel-Crafts (F.C.) alkylation of indoles and they involve chiral metal-complexes catalyzed reactions [5-7], enantioselective organocatalytic reactions via iminium ions [8,9] and chiral Bronsted Acids [10,11]. 
With regards to chiral Bronsted Acids, good efficiency but rather poor levels of enantioselectivity were observed in the F.C. alkylation of indoles with chalcones when a camphor-based Bronsted acid was used [10], while improved enantiomeric excesses (up to $56 \%$ e.e.) were obtained through the use of the $\mathrm{H}_{8}$-BINOL-based phosphoric acid of type $\mathbf{1}$ [11,12] $\left(\mathrm{R}=4-\mathrm{ClC}_{6} \mathrm{H}_{4}\right)(\mathrm{Scheme} 1$, Figure 1).

Scheme 1. Friedel-Crafts addition of Indole to Chalcones.<smiles>c1ccc2[nH]ccc2c1</smiles><smiles>[R]c1ccc(/C=C/C(=O)[AlH2])c([R])c1</smiles>

4

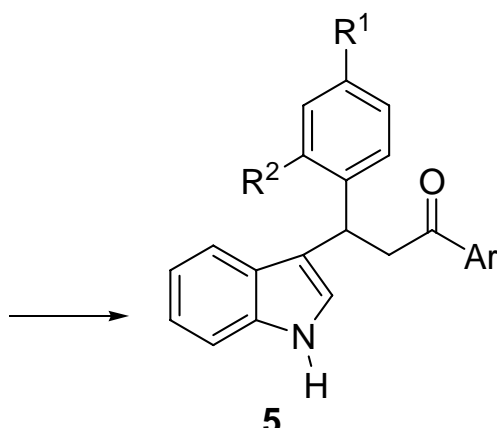

Figure 1. Chiral $\mathrm{H}_{8}$-BINOL-based phosphoric acid (S)-1 and BINOL-phosphoric acid (R)-2.<smiles>[R]C1=CC2CCCCC2C(c2c3c(cc([R])c2OP(=O)(O)O)CCCC3)=C1</smiles>

$(S)-1$<smiles>[R]c1cc2ccccc2c(-c2c(OP(=O)(O)O)c([R])cc3ccccc23)c1OP(=O)(O)O</smiles>

$(R)-2$

Notably, the use of a variety of BINOL-derived Bronsted Acid of type $(\boldsymbol{R})-2\left(\mathrm{R}=\mathrm{Ph}_{2} \mathrm{PO}, \mathrm{Ph}\right.$, 4- $\mathrm{ClC}_{6} \mathrm{H}_{4}, 4-\mathrm{MeC}_{6} \mathrm{H}_{4}, 4-\mathrm{PhC}_{6} \mathrm{H}_{4}, 2$-naphthyl, 3,5- $\left(\mathrm{F}_{3} \mathrm{C}\right)_{2} \mathrm{C}_{6} \mathrm{H}_{3}, 2,4,6-\mathrm{tBu}_{3} \mathrm{C}_{6} \mathrm{H}_{2}$ ) gave good yields (up to $75 \%)$ but rather lower e.e.s (2-35\% e.e.).

Taking into account that the different steric and electronic effects of the above cited substituents were found exert a deep influence, both on efficiency and enantioselectivity, we decided to investigate the catalytic properties of the BINOL-derivatives $2 \mathbf{a}\left(\mathrm{R}=\mathrm{SiPh}_{3}\right)$, and $\mathbf{2 b}\left(\mathrm{R}=4-\mathrm{NO}_{2} \mathrm{C}_{6} \mathrm{H}_{4}\right)$, bearing substituents with different electronic and steric properties, in the F.C. alkylation of indole with chalcones.

\section{Results and Discussion}

Initially chalcone 4a $\left(\mathrm{R}^{1}=\mathrm{R}^{2}=\mathrm{H} ; \mathrm{Ar}=\mathrm{Ph}\right)$ was chosen as a representative substrate and was submitted to reaction with indole 3 under the conditions reported in Table 1 and Scheme 2. Based on the results reported in Table 1, dichloromethane proved a superior solvent with respect to toluene (compare entries 1 and 2), while the organocatalyst $\mathbf{2} \mathbf{b}$ gave better results than $\mathbf{2 a}$, both in terms of yield and enantioselectivity, provided that more dilute solutions of chalcones 4 were used (compare entries 3, 4 and 5). 
Scheme 2. Asymmetric organocatalytic F.C. alkylation of indole 3 with chalcone $4 a$ catalyzed by $\mathbf{2 a}$ and $\mathbf{2} \mathbf{b}$.

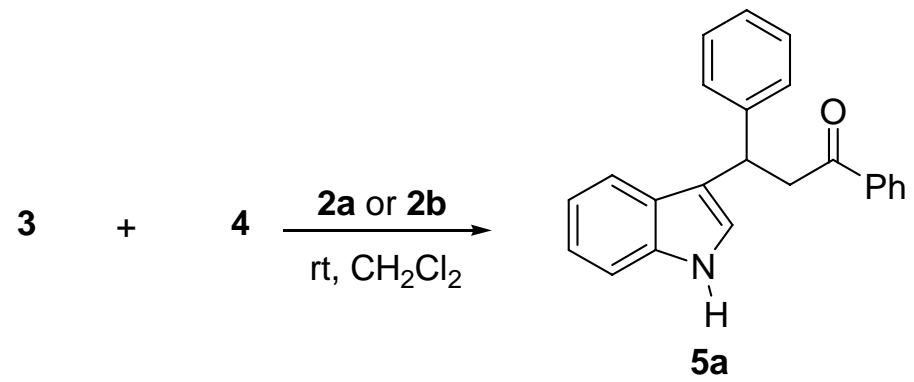

Table 1. Asymmetric organocatalytic F.C. alkylation of indole 3 with chalcone $\mathbf{4 a}$.

\begin{tabular}{llccc}
\hline Entry & Cat. 2 & Reac.Time/h & Yield (\%) $^{\mathbf{a}}$ & e.e.(\%) $^{\mathbf{b}}$ \\
\hline $1^{\text {c,d }}$ & 2a (0.05) & 24 & 25 & 40 \\
$2^{\text {e }}$ & 2a (0.05) & 48 & 25 & 49 \\
$3^{\text {d }}$ & 2a (0.1) & 24 & 35 & 33 \\
$4^{\text {d }}$ & 2b $(0.1)$ & 24 & 60 & 31 \\
$5^{\text {e }}$ & 2b $(0.1)$ & 48 & 82 & 52 \\
$6^{\text {e }}$ & 2b $(0.05)$ & 71 & 40 & 48 \\
$7^{\text {e }}$ & 2b $(0.02)$ & 120 & 25 & 46 \\
\hline
\end{tabular}

a All the yields refer to isolated chromatographically pure compounds whose structures were confirmed by analytical and spectroscopic data; ${ }^{b}$ Enantiomeric excess were determined by chiral HPLC; ${ }^{\mathrm{c}}$ In entry 1 toluene was used as solvent; ${ }^{\mathrm{d}} 0.5 \mathrm{M}$ solution of chalcones 4 was used; ${ }^{\mathrm{e}} 0.15 \mathrm{M}$ solution of chalcones 4 was used.

A lower organocatalyst loading (entries 6 and 7) caused a dramatic drop of the yields and a slight decrease of the e.e.s. It has to be noted that the e.e. of compound $5 \mathbf{a}$, obtained in entry 5 ( $52 \%$ e.e.) could be enhanced significantly $\left(72 \%\right.$ e.e.) by one only crystallization from $\mathrm{Et}_{2} \mathrm{O}$. The general scope of the procedure was then checked by submitting indole $\mathbf{3}$ to treatment with a set of chalcones $\mathbf{4}$ under the optimized conditions of entry 5 , Table 1 .

Scheme 3. Asymmetric F.C. addition of indole 3 to variously substituted chalcones 4 catalyzed by $\mathbf{2 b}$.<smiles>[R]c1ccc(/C=C/C(=O)[Al])c([R])c1</smiles>

As reported in Table 2, the alkylation of indole was found to take place in moderate to high yields (up to 98\%) with variously substituted chalcones while a moderate level of enantioselectivity could be observed for most of the reported starting materials. However, and very interestingly, in several cases the e.e.s of the Michael-type adducts 5 could be again enhanced noticeably (up to 98\%) by recrystallization. More simple $\alpha, \beta$-unsaturated ketones, such as benzylidene acetone, gave much less 
satisfactory results since the corresponding alkylation product was isolated in only $15 \%$ yield and $30 \%$ e.e.

Table 2. Asymmetric F.C. addition of indole 3 to variously substituted chalcones 4.

\begin{tabular}{ccccccc}
\hline Entry & $\mathbf{A r}$ & $\mathbf{R}^{\mathbf{1}}$ & $\mathbf{R}^{\mathbf{2}}$ & Product & $\mathbf{Y i e l d ( \% )}^{\mathbf{a}}$ & $\mathbf{E e ( \% )} \mathbf{b , \mathbf { c }}$ \\
\hline 1 & $\mathrm{Ph}$ & $\mathrm{H}$ & $\mathrm{H}$ & $\mathbf{5 a}$ & $82(40)^{\mathrm{d}}$ & $52(72)$ \\
2 & $\mathrm{Ph}$ & $\mathrm{H}$ & $\mathrm{Cl}$ & $\mathbf{5 b}$ & 98 & 48 \\
3 & $\mathrm{Ph}$ & $\mathrm{Me}$ & $\mathrm{H}$ & $\mathbf{5 c}$ & $58(43)^{\mathrm{d}}$ & $52(70)$ \\
4 & $\mathrm{Ph}$ & $\mathrm{OMe}$ & $\mathrm{H}$ & $\mathbf{5 d}$ & 36 & 41 \\
5 & $\mathrm{Ph}$ & $\mathrm{NO}_{2}$ & $\mathrm{H}$ & $\mathbf{5 e}$ & 77 & 46 \\
6 & $\mathrm{Ph}$ & $\mathrm{Cl}$ & $\mathrm{H}$ & $\mathbf{5 f}$ & $60(30)^{\mathrm{d}}$ & $52(98)$ \\
7 & $4-\mathrm{ClC}_{6} \mathrm{H}_{4}$ & $\mathrm{H}$ & $\mathrm{H}$ & $\mathbf{5 g}$ & $65(33)^{\mathrm{d}}$ & $54(97)$ \\
8 & $4-\mathrm{MeC}_{6} \mathrm{H}_{4}$ & $\mathrm{H}$ & $\mathrm{H}$ & $\mathbf{5 h}$ & 44 & 46 \\
9 & $4-\mathrm{NO}_{2} \mathrm{C}_{6} \mathrm{H}_{4}$ & $\mathrm{OMe}$ & $\mathrm{H}$ & $\mathbf{5 i}$ & $73(47)^{\mathrm{d}}$ & $42(51)$ \\
\hline
\end{tabular}

${ }^{\text {a }}$ All the yields refer to isolated chromatographically pure compounds whose structures were confirmed by analytical and spectroscopic data; ${ }^{b}$ Enantiomeric excess were determined by chiral HPLC; ${ }^{c}$ Values in parentheses represent the enantiomeric excess observed after crystallization of 5a and 5c (from $\mathrm{Et}_{2} \mathrm{O}$ ), $5 \mathbf{f}$ and $\mathbf{5 g}$ (from $\mathrm{CH}_{2} \mathrm{Cl}_{2} /$ light petroleum ether) and $5 \mathrm{i}$ (from $\mathrm{Et}_{2} \mathrm{O}$ /light petroleum ether); ${ }^{\mathrm{d}}$ Values in parentheses represent the yield after crystallization.

\section{Experimental}

\subsection{General}

All chemicals were purchased from Sigma-Aldrich and used without any further purification. TLC was performed on silica gel $60 \mathrm{~F}_{254} 0.25 \mathrm{~mm}$ on glass plates (Merck) and non-flash chromatography was performed on silica gel (0.063-0.200 mm) (Merck). All ${ }^{1} \mathrm{H}$ - and ${ }^{13} \mathrm{C}-\mathrm{NMR}$ spectra were recorded with a DRX $400 \mathrm{MHz}$ Bruker instrument (400.135 MHz for ${ }^{1} \mathrm{H}$ and $100.03 \mathrm{MHz}$ for ${ }^{13} \mathrm{C}$ ), using $\mathrm{CDCl}_{3}$ ( $\delta=7.26 \mathrm{ppm}$ in ${ }^{1} \mathrm{H}-\mathrm{NMR}$ spectra and $\delta=77.0 \mathrm{ppm}$ in ${ }^{13} \mathrm{C}$-NMR spectra) as solvent. ${ }^{1} \mathrm{H}$ data are reported as follows: chemical shift ( $\delta$ in ppm), multiplicity (s singlet, $d$ doublet, t triplet, dd doublet of doublets, $\mathrm{m}$ multiplet) and coupling costant $(J$ in Hz). Optical rotations were measured on a JASCO DIP-1000 polarimeter operating at the sodium D line at room temperature. Concentration is given in $\mathrm{g} / 100 \mathrm{~mL}$. IR spectra were recorded on a Bruker spectrometer. The HPLC analyses were performed with Waters Associates equipment (Waters 2487 Dual $\lambda$ absorbance Detector) using a CHIRALPAK AD-H column with hexane/isopropyl alcohol mixtures (composition and flow rate as indicated). HPLC methods were calibrated with the corresponding racemic mixtures. Mass spectrometry analysis was carried out using an Waters 4 micro quadrupole electrospray spectrometer. The elemental analyses were calculated with FLASH EA 1112 Thermo equipment. Melting points were determined with an Electrothermal 9100 apparatus. The known compounds have been identified by comparison of spectral data with those reported [8,11].The absolute configureurations of the optically active compounds 5a was determined on the basis of the measured optical rotation compared with literature values $[8,11]$. 


\subsection{Typical experimental procedure}

To a mixture of chalcone $(0.125 \mathrm{mmol})$ and catalyst $(0.0125 \mathrm{mmol}) 1.2 \mathrm{eq}$. of indole $(0.15 \mathrm{mmol})$ were added and stirred in dry dichloromethane $(0.75 \mathrm{~mL})$ at room temperature. The reaction was monitored by TLC analysis. After 48 hours a saturated aqueous $\mathrm{NaHCO}_{3}$ solution $(0.75 \mathrm{~mL})$ was added dropwise and the organic layer was extracted in $\mathrm{CH}_{2} \mathrm{Cl}_{2}$, dried over $\mathrm{MgSO}_{4}$ and concentrated in vacuo. The residue was purified by column chromatography on silica gel in gradient elution with petroleum pther/ethyl acetate to give the pure product.

3-(1H-Indol-3-yl)-1-phenyl-3-p-tolylpropan-1-one (5c): Yellow solid $m / z 340\left[\mathrm{M}+\mathrm{H}^{+}\right], 362\left[\mathrm{M}+\mathrm{Na}^{+}\right]$, $378\left[\mathrm{M}+\mathrm{K}^{+}\right]$; M.p. 91-92 ${ }^{\circ} \mathrm{C}$; IR (KBr, neat) 3418, 2918, 1687; $[\alpha]_{\mathrm{D}}=\left(\mathrm{CHCl}_{3} \mathrm{c}=0.65,52 \%\right.$ e.e. $)$ $=-11.6$; HPLC analysis: hexane $/ i-\mathrm{PrOH} 98: 2$, flow rate $0.7 \mathrm{~mL} / \mathrm{min} . \mathrm{t}_{\mathrm{R}}($ major $)=97.3, \mathrm{t}_{\mathrm{R}}($ minor $)=$ 100.3 min.; ${ }^{1} \mathrm{H}-\mathrm{NMR}$ : $\delta 7.97(1 \mathrm{H}, \mathrm{bs}), 7.94(2 \mathrm{H}, \mathrm{d}, J=7.4 \mathrm{~Hz}), 7.54(1 \mathrm{H}, \mathrm{t}, J=7.2 \mathrm{~Hz}), 7.47-7.40(3 \mathrm{H}$, m), $7.31(1 \mathrm{H}, \mathrm{d}, J=8.1 \mathrm{~Hz}), 7.25(2 \mathrm{H}, \mathrm{d}, J=7.3 \mathrm{~Hz}), 7.15(1 \mathrm{H}, \mathrm{t}, J=7.2 \mathrm{~Hz}), 7.08-6.97(4 \mathrm{H}, \mathrm{m}), 5.04$ $(1 \mathrm{H}, \mathrm{t}, J=7.2 \mathrm{~Hz}), 3.82(1 \mathrm{H}, \mathrm{dd}, J=16.6 ; 6.8 \mathrm{~Hz}), 3.71(1 \mathrm{H}, \mathrm{d}, J=16.6 ; 7.6 \mathrm{~Hz}), 2.28(3 \mathrm{H}, \mathrm{s})$; ${ }^{13}$ C-NMR: $\delta$ 199.2, 141.7, 137.7, 137.2, 136.2, 133.5, 129.6, 129.1, 128.6, 128.2, 127.2, 122.6, 121.9, 120, 111.6, 45.8, 38.4, 21.5; Anal. Calcd for $\mathrm{C}_{24} \mathrm{H}_{21} \mathrm{NO} \mathrm{C}, 84.92 ; \mathrm{H}, 6.24 ; \mathrm{N}, 4.13$; found $\mathrm{C}, 84.50 ; \mathrm{H}$, $6.10 ; \mathrm{N} 4.10$.

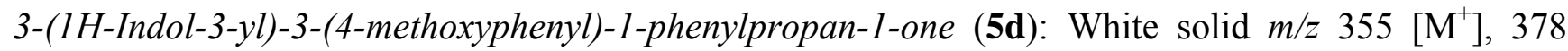
$\left[\mathrm{M}+\mathrm{Na}^{+}\right], 394\left[\mathrm{M}+\mathrm{K}^{+}\right]$; M.p. $116-118^{\circ} \mathrm{C}$; IR (KBr, neat) 3424, 2918, 1677, 1180; $[\alpha]_{\mathrm{D}}=\left(\mathrm{CHCl}_{3}\right.$ $\mathrm{c}=0.33,41 \%$ e.e. $\%)=-12.3$; HPLC analysis: hexane $/ i$-PrOH 80:20 flow rate, $0.9 \mathrm{~mL} / \mathrm{min}$. $\mathrm{t}_{\mathrm{R}}$ (major) $=24.2, \mathrm{t}_{\mathrm{R}}($ minor $)=26.05 \mathrm{~min}$; ${ }^{1} \mathrm{H}-\mathrm{NMR}: \delta 7.98(1 \mathrm{H}, \mathrm{bs}) 7.93(2 \mathrm{H}, \mathrm{d}, J=7.2 \mathrm{~Hz}), 7.54(1 \mathrm{H}, \mathrm{t}$, $J=7.4 \mathrm{~Hz}), 7.44-7.41(2 \mathrm{H}, \mathrm{m}), 7.31(1 \mathrm{H}, \mathrm{d}, J=8.0 \mathrm{~Hz}), 7.26(2 \mathrm{H}, \mathrm{d}, J=8.6 \mathrm{~Hz}), 7.14(1 \mathrm{H}, \mathrm{t}, J=7.2$ $\mathrm{Hz}), 7.04-6.98(2 \mathrm{H}, \mathrm{m}), 6.79(2 \mathrm{H}, \mathrm{d}, J=8.6 \mathrm{~Hz}), 5.02(1 \mathrm{H}, \mathrm{t}, J=7.2 \mathrm{~Hz}), 3.79(1 \mathrm{H}, \mathrm{dd}, J=16.6 ; 6.6$ $\mathrm{Hz}), 3.75(3 \mathrm{H}, \mathrm{s}), 3.69(1 \mathrm{H}, \mathrm{dd}, J=16.6 ; 7.8 \mathrm{~Hz}) ;{ }^{13} \mathrm{C}-\mathrm{NMR}: \delta$ 199.2, 157.9, 137.7, 137.2, 136.8, 133.5, 129.3, 129.1,128.6, 122.7, 121.8, 120.1, 119.9, 114.3, 111.6, 55.7, 45.9, 38.0; Anal. Calcd for $\mathrm{C}_{24} \mathrm{H}_{21} \mathrm{NO}_{2} \mathrm{C}, 81.10 ; \mathrm{H}, 5.96 ; \mathrm{N}, 3.94$; found $\mathrm{C}, 81.05 ; \mathrm{H}, 5.89 ; \mathrm{N}, 3.90$.

3-(4-Chlorophenyl)-3-(1H-indol-3-yl)-1-phenylpropan-1-one (5f): White solid $\mathrm{m} / \mathrm{z} 359\left[\mathrm{M}^{+}\right], 382$ $\left[\mathrm{M}+\mathrm{Na}^{+}\right]$; M.p. $98-99^{\circ} \mathrm{C}$; IR (KBr, neat) 3445, 2916, 1694, 1219; $[\alpha]_{\mathrm{D}}=\left(\mathrm{CHCl}_{3} \mathrm{c}=0.13,52 \%\right)=-4.6$; HPLC analysis: hexane $/ i$-PrOH 80:20, flow rate $0.8 \mathrm{~mL} / \mathrm{min}, \mathrm{t}_{\mathrm{R}}$ (major) $=16.9, \mathrm{t}_{\mathrm{R}}$ (minor) $=18.9 \mathrm{~min}$; ${ }^{1} \mathrm{H}-\mathrm{NMR}: \delta 8.01(1 \mathrm{H}, \mathrm{bs}), 7.93(2 \mathrm{H}, \mathrm{d}, J=7.1 \mathrm{~Hz}), 7.57-6.98(11 \mathrm{H}, \mathrm{m}), 5.05(1 \mathrm{H}, \mathrm{t}, J=7.4 \mathrm{~Hz}), 3.81$ $(1 \mathrm{H}, \mathrm{dd}, J=16.8 ; 6.4 \mathrm{~Hz}), 3.69(1 \mathrm{H}, \mathrm{dd}, J=16.8,8.0 \mathrm{~Hz}) ;{ }^{13} \mathrm{C}-\mathrm{NMR}: \delta 198.7,138.3,136.5,133.7$, 131.5, 129.7, 129.2, 129.0, 128.6, 124.1, 122.9, 122.6, 121.8, 120.1, 119.9, 111.7, 45.5, 38.1; Anal. Calcd for $\mathrm{C}_{23} \mathrm{H}_{18} \mathrm{CINO}$ C, 84.89; H, 5.89; N, 4.30; found C, 84.50; H, 5.70; N, 4.25.

1-(4-Chlorophenyl)-3-(1H-indol-3-yl)-3-phenylpropan-1-one (5g): White solid m/z $360\left[\mathrm{M}+\mathrm{H}^{+}\right], 382$ $\left[\mathrm{M}+\mathrm{Na}^{+}\right], 398\left[\mathrm{M}+\mathrm{K}^{+}\right]$; M.p. 101-102 ${ }^{\circ} \mathrm{C}$; IR (KBr, neat) 3421, 2922, 1685, 1093; $[\alpha]_{\mathrm{D}}=\left(\mathrm{CHCl}_{3}\right.$ $\mathrm{c}=0.33,54 \%)=-18$; HPLC analysis: hexane $/ i$-PrOH 80:20, flow rate $0.8 \mathrm{~mL} / \mathrm{min} . \mathrm{t}_{\mathrm{R}}$ (major) $=19.9, \mathrm{t}_{\mathrm{R}}$ $($ minor $)=22.3 \mathrm{~min} ;{ }^{1} \mathrm{H}-\mathrm{NMR}: \delta 7.99(1 \mathrm{H}, \mathrm{bs}), 7.85(2 \mathrm{H}, \mathrm{d}, J=8.4 \mathrm{~Hz}), 7.45-6.96(10 \mathrm{H}, \mathrm{m}), 5.05(1 \mathrm{H}$, t, $J=7.2 \mathrm{~Hz}), 3.78(1 \mathrm{H}, \mathrm{dd}, J=16.6 ; 6.8 \mathrm{~Hz}), 3.69(1 \mathrm{H}, \mathrm{dd}, J=16.6 ; 7.7 \mathrm{~Hz}) ;{ }^{13} \mathrm{C}-\mathrm{NMR}: \delta 198.0$, 
146.5, 139.9, 137.1, 135.9, 130.0, 129.4, 129.0, 128.3, 126.9, 122.7, 121.9, 120.0, 119.0, 111.7, 45.6, 38.8. Anal. Calcd. for $\mathrm{C}_{23} \mathrm{H}_{18} \mathrm{ClNO}$, $84.89 ; \mathrm{H}, 5.89 ; \mathrm{N}, 4.30$; found $\mathrm{C}, 84.60 ; \mathrm{H}, 5.75 ; \mathrm{N}, 4.28$.

3-(1H-Indol-3-yl)-3-phenyl-1-p-tolylpropan-1-one (5h): White solid $m / z 340\left[\mathrm{M}^{+} \mathrm{H}^{+}\right], 362\left[\mathrm{M}+\mathrm{Na}^{+}\right]$; M.p. $167-169^{\circ} \mathrm{C}$; IR (KBr, neat) $3419,2918,1704,1181 ;[\alpha]_{\mathrm{D}}=\left(\mathrm{CHCl}_{3} \mathrm{c}=0.33,46 \%\right)=-19$; $\mathrm{HPLC}$ analysis: hexane $/ \mathrm{i}-\mathrm{PrOH}$ 90:10, flow rate $1.0 \mathrm{~mL} / \mathrm{min} . \mathrm{t}_{\mathrm{R}}$ (major) $=38.1, \mathrm{t}_{\mathrm{R}}$ (minor) $=45.4 \mathrm{~min}$; ${ }^{1} \mathrm{H}-\mathrm{NMR}: \delta 7.97(1 \mathrm{H}, \mathrm{bs}), 7.84(2 \mathrm{H}, \mathrm{d}, J=8.0 \mathrm{~Hz}), 7.44-6.99(12 \mathrm{H}, \mathrm{m}), 5.07(1 \mathrm{H}, \mathrm{t}, J=7.1 \mathrm{~Hz}), 3.79$ $(1 \mathrm{H}, \mathrm{dd}, J=16.6 ; 6.9 \mathrm{~Hz}), 3.69(1 \mathrm{H}, \mathrm{dd}, J=16.6 ; 7.6 \mathrm{~Hz}), 2.39(3 \mathrm{H}, \mathrm{s}) ;{ }^{13} \mathrm{C}-\mathrm{NMR}: \delta 198.7$, $144.3,143.9,135.2,133.9,129.7,128.9,128.7,128.3,126.7,122.6,121.9,120.1,119.9,111.6,45.6$, 38.8, 22.1; Anal. Calcd for $\mathrm{C}_{24} \mathrm{H}_{21} \mathrm{NO}$ C, 84.92; H, 6.24; N, 4.13; found C, 84.80; H, 6.20; N, 4.10.

\section{Conclusions}

In conclusion, we have developed a Michael-type reaction of indole leading to variously substituted chalcones by using chiral Bronsted Acid $\mathbf{2 b}$ as catalyst. The reaction proceeds with good efficiency and moderate enantioselectivity. The possibility to obtain the alkylation products in much higher e.e.s (up to $98 \%$ ) after only a single recrystallization provides a practical method to synthesize highly enantiopure 2-indole derivatives.

\section{Acknowledgements}

We are grateful to MIUR and University of Salerno for financial support.

\section{References and Notes}

1. Saxton, J.E. Recent progress in the chemistry of the monoterpenoid indole alkaloids. Nat. Prod. Rep. 1997, 14, 559-590.

2. Toyota, M.; Ihara, N. Recent progress in the chemistry of non-monoterpenoid indole alkaloids. Nat. Prod. Rep. 1998, 15, 327-340.

3. Jorgensen, K.A. Asymmetric Friedel-Crafts reactions: catalytic enantioselective addition of aromatic and heteroaromatic $\mathrm{C}-\mathrm{H}$ bonds to activated alkenes, carbonyl compounds, and imines. Synthesis 2003, 1117-1125.

4. Bandini, M.; Melloni, A.; Umani-Ronchi, A. New catalytic approaches in the stereoselective Friedel-Crafts alkylation reaction. Angew. Chem. Int. Ed. 2004, 43, 550-556.

5. Bandini, M.; Fagioli, M.; Melchiorre, P.; Melloni, A.; Umani-Ronchi, A. Catalytic enantioselective conjugate addition of indoles to simple $\alpha, \beta$-unsaturated ketones. Tetrahedron Lett. 2003, 44, 5843-5846.

6. Blay, G.; Fernandez, I.; Pedro, J.R.; Vila, C. Highly enantioselective Friedel-Crafts alkylations of indoles with simple enones catalyzed by Zirconium(IV)-BINOL complexes. Org. Lett. 2007, 9, 2601-2604.

7. Jensen, K.B.; Thorhauge, J.; Hazell, R.G.; Jorgensen, K.A. Catalytic asymmetric Friedel-Crafts alkylation of $\beta, \gamma$-unsaturated $\alpha$-ketoesters: enantioselective addition of aromatic $\mathrm{C}-\mathrm{H}$ bonds to alkenes. Angew. Chem. Int. Ed. 2001, 40, 160-163. 
8. Bartoli, G.; Bosco, M.; Carlone, A.; Pesciaioli, F.; Sembri, L.; Melchiorre, P. Organocatalytic asymmetric Friedel-Crafts alkylation of indoles with simple $\alpha, \beta$-unsaturated ketones. Org. Lett. 2007, 9, 1403-1405.

9. Chen, W.; Du, W.; Yue, L.; Li, R.; Wu, Y.; Ding, L.S.; Chen, Y.C. Organocatalytic enantioselective indole alkylation of $\alpha, \beta$-unsaturated ketones. Org. Biomol. Chem. 2007, 5, 816-821.

10. Zhou, W.; Xu, L.W.; Li, L.; Yang.; Xia, C.G. Enantioselective Michael-Type Friedel-Crafts Reactions of Indoles to Enones Catalyzed by a Chiral Camphor-Based Bronsted. Eur. J. Org. Chem. 2006, 5225-5227.

11. Thang, H.Y.; Lu, A.D.; Zhou, Z.H.; Zhao, G.F.; He, L.N.; Thang, C.C. Chiral Phosphoric Acid Catalyzed Asymmetric Friedel-Crafts Alkylation of Indoles with Simple $\alpha, \beta$-Unsaturated Aromatic Ketones. Eur. J. Org. Chem. 2008, 1406-1410.

12. For a review on enantioselective $\mathrm{C}-\mathrm{C}$ bond forming reaction catalyzed by BINOL-derived phosphoric acid: Terada, M. Binaphthol-derived phosphoric acid as a versatile catalyst for enantioselective carbon-carbon bond forming reactions. Chem. Commun. 2008, 35, 4097-4112.

Sample Availability: Samples of the compounds are available from the authors.

(C) 2009 by the authors; licensee Molecular Diversity Preservation International, Basel, Switzerland. This article is an open-access article distributed under the terms and conditions of the Creative Commons Attribution license (http://creativecommons.org/licenses/by/3.0/). 